\title{
BioFeature
}

\section{Bioinformatics: Use in Bacterial Vaccine Discovery}

BioTechniques 31:636-659(September 2001)

\author{
Robert J. Zagursky and \\ David Russell ${ }^{1}$ \\ Wyeth Lederle Vaccines, \\ West Henrietta, NY, and ${ }^{1}$ Idea \\ Integration, Fairport, NY, USA
}

\section{INTRODUCTION}

Bioinformatics is a name coined in the 1990s in response to the increase in biological information and the ease of sharing this information using the Internet. Bioinformatics is defined as "a scientific discipline that encompasses all aspects of biological information acquisition, processing, storage, distribution, analysis, and interpretation that combines the tools and techniques of mathematics, computer science, and biology with the aim of understanding the biological significance of a variety of data" (14). Hence, bioinformatics covers a multiple of scientific disciplines and encompasses various areas of genomic analysis, including: (i) computational genomics (the storage and analysis of DNA sequence using various software), (ii) proteomics (laboratory methods used to determine what proteins are actually expressed and possible cellular location), (iii) transcriptional profiling (the analysis of mRNAs at different stages of cell growth and conditions), (iv) functional genomics (combined function prediction and experimental biological analysis of gene function), and ( $v$ ) structurefunction determinations (predicted as well as determined 3-D structures and comparison to known protein structures and functions). Figure 1 shows the integration of these various scientific disciplines used by pharmaceutical companies in their attempt to help identify drug and vaccine targets. This review focuses on how bioinformatics, specifically computational genomics, is being applied in the search for protein subunit vaccines against bacterial diseases. (For a general review on bioinformatics, see Reference 100.)

Even today, with the best of medicines available, the leading cause of death in young children, worldwide, is infectious disease, as shown in Figure 2. Preventing infection or limiting the effects of the infection by vaccination dates back to the first scientific study and development of a safe vaccine by Jenner in 1796. Jenner utilized a live cowpox virus to vaccinate humans against smallpox virus. Today, there are many safe human vaccines directed toward preventing disease caused by various infectious agents (61). Present-day man is fortunate to live in a world free of smallpox and nearly free of polio thanks to the World Health Organization's (WHO) efforts of trying to eradicate diseases by vaccination. Advances in vaccine development have often been the result of applying basic research discoveries. For example, multivalent vaccines comprised of different capsular polysaccharides from Streptococcus pneumoniae, one of the major causes of bacterial meningitis, have been in use since 1946 and prevent bacteremic disease in older children and adults. Yet, these same vaccines were found to be largely ineffective in children less than two years of age and of variable effectiveness in children less than five years of age because of the immaturity of their immune systems (40). The capsular polysaccharides, which are also non-immunogenic in infants, became highly effective vaccine components when conjugated with protein antigens (37). Using glycoconjugate technology, an effective vaccine for prevention of invasive pneumococ- 
cal disease in infants has recently been developed (15). This vaccine, licensed in early 2000, provides approximately $100 \%$ protection against the seven most prevalent serotypes of $S$. pneumoniae.

A multivalent vaccine to prevent bacterial meningitis and sepsis caused by Neisseria meningitidis is also needed. In the case of this pathogen, there are at least 13 different serogroups, based on the bacterial capsular polysaccharide composition, with the majority of the disease caused by groups A, B, C, Y, and W-135 (61). Polysaccharidebased vaccines are available (for older children and adults) against serogroups A, C, Y, and W-135, and a serogroup C conjugate vaccine is available in several European countries. Unfortunately, the serogroup B capsular polysaccharide is immunobiologically similar to polysialic acid expressed on human cells and is poorly immunogenic in humans. Thus, trying to generate antibodies to this polysaccharide could potentially induce autoimmune disease and harm the vaccinee. Therefore, a protein antigenbased vaccine is being pursued (102).

As indicated above, several existing vaccines rely on generating an effective immune response against the bacterial polysaccharide capsule. Other effective vaccines are directed toward bacterial toxins (e.g., diphtheria, tetanus). For organisms that make no capsular polysaccharide or do not produce toxin-mediated diseases, other targets have to be identified. The most promising candi- dates are surface-exposed or secreted proteins. In the "pre-genomic sequencing era", a common approach used in early vaccine discovery programs was to generate polyclonal or monoclonal antisera in animals by administering either live or inactivated bacteria. The sera were analyzed in an immuno-assay format against either whole bacteria or a bacterial protein preparation. The major reactive proteins, usually less than a dozen, were further characterized by purifying the native proteins and analyzing the subsequent sera, generated from vaccinating animals with the same proteins. The sera were tested in functional immunobiological assays and then in an animal model. The proteins that elicited functional immunobiological activity

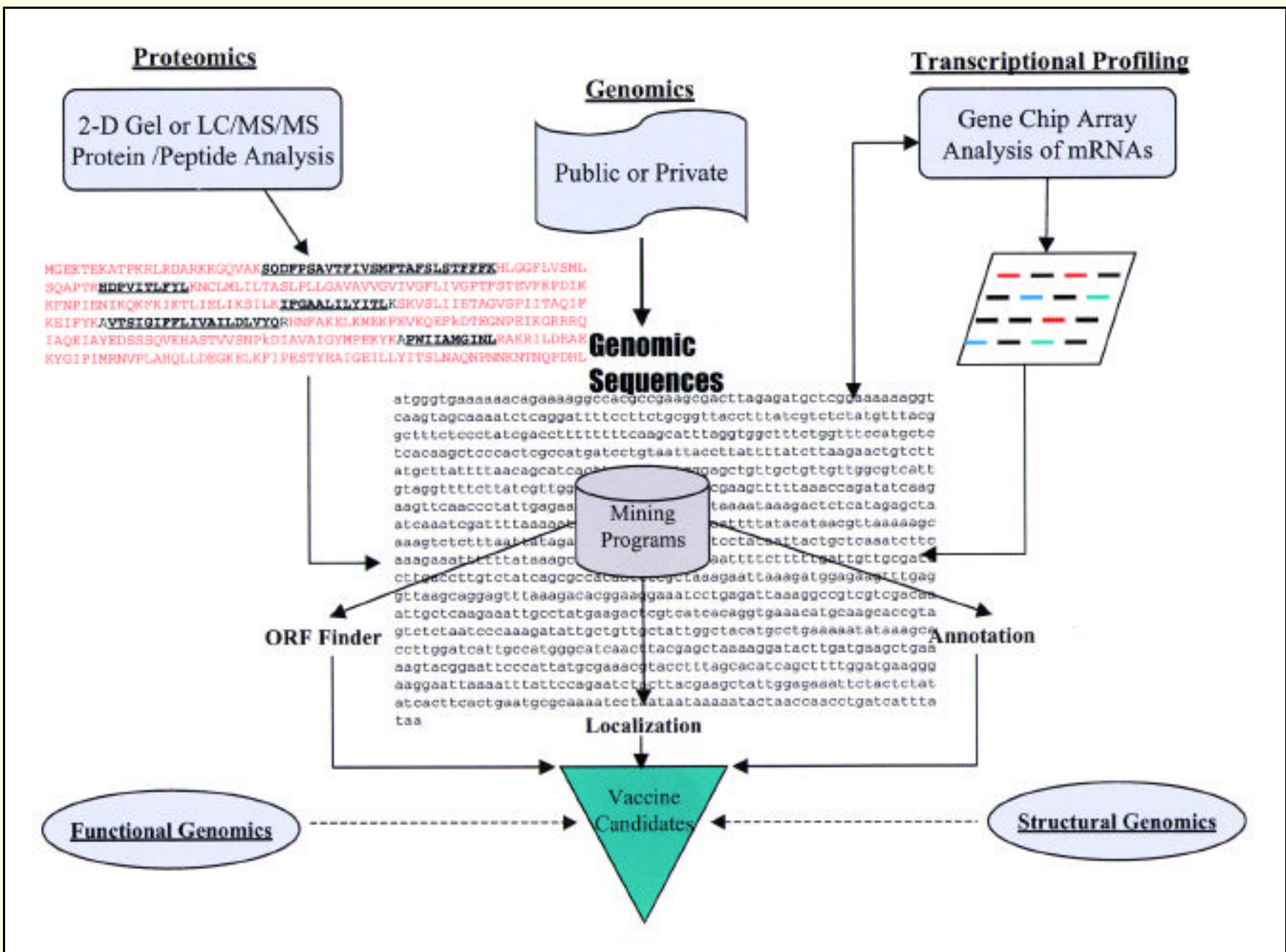

Figure 1. Various research disciplines of bioinformatics. The center of bioinformatics is genomic DNA sequence that feeds into various research disciplines. Genomics mines the sequence and predicts various features such as genes and their putative function. Limited protein sequence generated by proteomics is searched through the translated genomic sequence for gene identification. Transcriptional profiling is dependent on genomics for gene identification and subsequent chip design. Structural genomics predictions are based on protein sequence and comparison with various structural databases. Functional genomics helps to correctly define gene function using both computational and biological information. 
were taken into the next phase of basic research. This second phase consisted of N-terminal amino acid sequencing, cloning and expression of the genes into a safe bacterial host, such as the wellknown laboratory strain, K12 of E. coli, and analyzing the purified engineered proteins in various functional immunobiological assays. The total time it took to reach the end of the second phase could be as short as six months or as long as several years. Today, if one has the genomic sequence of an organism, one can rapidly identify literally hundreds of potential vaccine candidates during the first phase of the study and rapidly clone the genes by PCR technology into various expression vectors. This approach has recently been termed "reverse vaccinology" (103). The bottleneck now seems to be in the testing of the large number of gene candidates. Newer technologies, such as the linear expressed elements DNA vaccination method, are being developed that should reduce this time (127).

\section{COMPUTATIONAL GENOMICS}

Today, in what one might consider being the "post-genomic sequencing era", there is a tremendous amount of raw sequence data and related information. The total amount of information available to a researcher is becoming "mind boggling" and may give one a feeling of data overload. In just six years since the first complete bacterial genome was sequenced (42), about 200 microbial genomes (about 40 complete) are being sequenced (see http://wit.integratedgenomics.com/GOLD/ and Reference 71). Having the genomic sequence of a bacterial organism allows one to search (mine) the genome and predict genes (protein and RNA), promoters and other regulatory sites, as well as the origin of replication and other features. To know the total genetic sequence of an organism was once thought to be the "Holy Grail" in genetics. However, understanding and deciphering all that information and assigning functions to the genes is turning out to be a long and exciting learning process for all genomes being sequenced. For vaccine discovery, it is most important to identify those genes, whether their function is known or not, that encode surface-exposed antigens. This is in contrast to drug discovery research in which knowledge of the function of a gene is paramount. Thus, of the predicted genes of a bacterial genome (gene density is roughly one gene per 1000 nucleotides-smallest genome, Mycoplasma genitalium, about $5.8 \mathrm{kbp}$; largest genome, Nostoc punctiforme about 7.5 Mbp), a screen of the genetic information for predicted surface or secreted proteins is done well before a researcher enters a wet laboratory. A schematic flowchart of the work progression toward having a vaccine candidate is shown in Figure 3. The first step in any mining operation is to identify the genes or open reading frames (ORFs) contained within the genomic sequence.

\section{ORF (Gene) Finding}

In the scientific literature today, an ORF and gene are being used interchangeably to define the region between two in-frame stop codons that may contain a putative start codon regardless of whether there is biological proof that the encoded protein even exists. A number of ORF finder programs exist today to identify genes in a prokaryotic genome. The main feature of many of these programs is the use of some type of "learning" software that is trained on a dataset. Based on the training dataset, a model is built that incorporates information about codon frequency and probability of a certain nucleotide or state following another nucleotide or state. (For theoretical overview on sequence models, see Reference 36.) This is done in both sequence directions of the DNA looking in all six reading frames. Some of the ORF finders invoke other features for predicting genes such as allowed amount of nucleotide overlap between two genes, the start and stop codons to be used, and whether a ribosomal binding site or Shine-Dalgarno box is present (116). One of the best-known programs is GeneMark (17), which uses a Markov chain model based on a training set of known genes and noncoding DNA regions extracted from an external database for learning. The training set and the sequence to be analyzed should be of a similar statistical composition for best results. An E. coli training set would not be effective in predicting genes from a completely

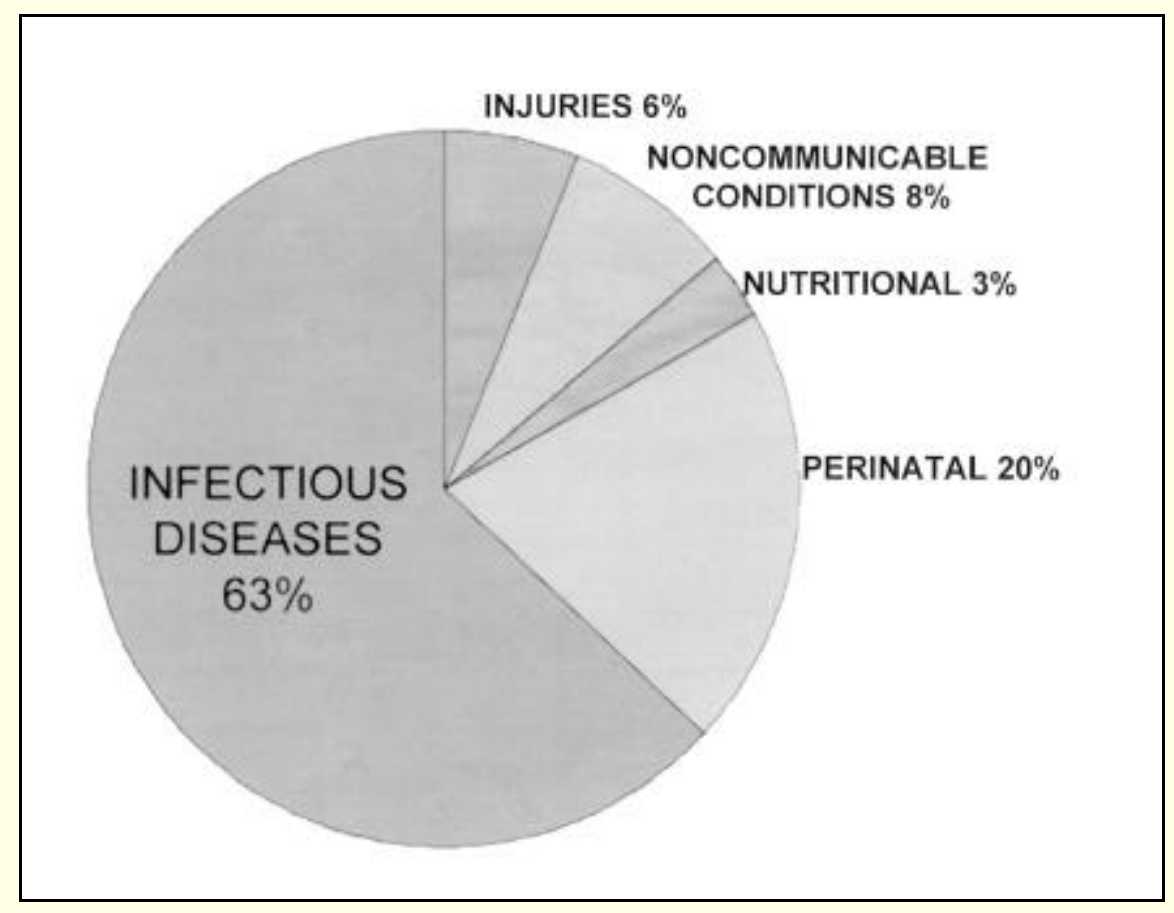

Figure 2. Causes of death in children worldwide. Distribution of main causes of death among children, ages 0-4 years - estimates are for 1998, worldwide. Data from the 1999 World Health Organization Report on Infectious Disease can be accessed at http://www.who.int/infectious-disease-report/index-rpt $99 . \mathrm{html}$. 
different species. A recent enhancement in this program uses a hidden Markov model (HMM) framework and RBS pattern to improve gene prediction (78). Another well-known and widely used program is GLIMMER, which was developed in part at The Institute for Genomic Research (TIGR) and uses a technique called interpolated Markov models (109) that trains itself on the genome being analyzed. GLIMMER first extracts large ORFs from the genome being studied as the training set and then uses an HMM trained on the extracted dataset to find genes. Other programs such as ORPHEUS use a DNA to protein database similarity search to derive statistical characteristics of ORFs (44), and SELFID, a program similar to GeneMark, that uses an $a b$ initio iterative Markov modeling procedure and can process unassem bled sequences (8). The accuracy of ORF finding can be as high as $99 \%$ based on current knowledge and the latest ORF finders (33). Ultimately, predictions need to be validated by laboratory experiments, and new ORFs will continually be found that were not pre- dicted by software. For instance, a database of new gene products characterized since the completion of the $E$. coli genome (16) is available at http://web. bham.ac.uk/bcm4ght6/res.html and is maintained by Gavin Thomas (131).

A number of factors can affect the true determination of an ORF such as the start and stop codons used. The major start codon is AUG, while GUG and UUG are minor codons. Rare start codons AUU, CUG, AAA, GGA, and UGC have also been found $(2,16)$. There are even cases when multiple proteins are encoded within the same gene that use the same stop codon but different start codons such as the $\operatorname{clp} A$ and $\operatorname{clpB}$ genes of $E$. coli $(95,114)$. Another example is the nolA gene from Bradyrhizpbium japonicum that produces three functionally distinct proteins (77). As in the case of start codons, there are a number of different stop codons (UAG, UAA, and UGA), but exceptions have been found both in mycoplasma and in some mitochondria. In these, UGA and UGG encode for tryptophan (65). The UGA codon can also direct the incorporation of the

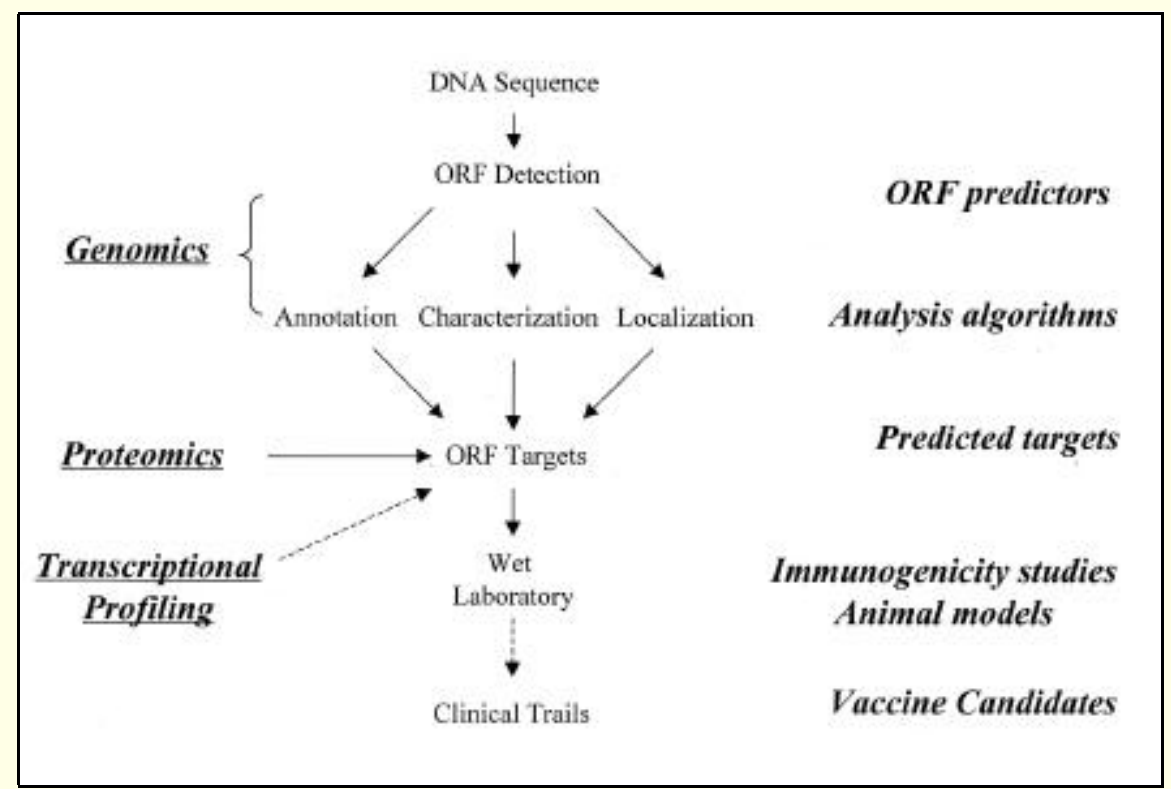

Figure 3. Flow diagram of vaccine discovery research involving bioinformatics. Starting at the top, ORFs are predicted from genomic DNA and further analyzed using various algorithms to help define function and location. Predicted surface-localized candidates are combined with experimentally determined surface proteins identified using proteomics, which results in a preliminary vaccine target list. From this list, the genes are then fed into the next phase of laboratory cloning, expression, and antibody generation. Successful gene candidates that make it through this phase are then tested in various pre-clinical immunological assays and animal model studies. Only those candidates that show positive results are further analyzed before going into clinical studies. Other research disciplines, such as transcriptional profiling, may contribute to this flow pathway.

modified amino acid selenocysteine, but, for UGA to be decoded (or recoded), additional elements must be present (75). Ribosomal -1 frameshifting can also account for extended protein sequences at the $3^{\prime}$ end of an ORF, such as in the case of the Bacillus subtilis $c d d$ gene (81). There are known cases of genes totally embedded within another gene but out-of-frame such as the Holin-like protein of Staphylcoccus aureus bacteriophage 187 (76) and the $R z l$ gene of $E$. coli bacteriophage $\lambda$ (55). The phenomenon of joining two ORFs by translational bypassing has been demonstrated for T4 gene 60 (58). Identification of an RBS in front of a potential start codon is helpful in defining an ORF (115). However, because the complementarity to the $3^{\prime}$ end of the $16 \mathrm{~S}$ rRNA does not have to be exact, computational methods for RBS identification are still imperfect. Computer analysis for finding RBS among various prokaryotic genomes does reveal a correlation between the RBS properties of a given genome and the potential gene start prediction accuracy $(43,54)$. However, there are cases when there is no detectable base pairing between the region upstream of start codons and the $3^{\prime}$ end of the $16 \mathrm{~S}$ rRNA (43). There are also cases of zero to just a few nucleotides of leader sequence upstream of the translation start codon. For example, there are zero nucleotides of $c I$ repressor gene of bacteriophage $\lambda$ transcribed from the Prm promoter (52) or the cre recombinase gene of bacteriophage $\mathrm{P} 1$ transcribed from the $\mathrm{p} R 3$ promoter (124), and there are two nucleotides from the bop gene in archaebacterium Halobacterium halobium (32). Finally, the size of the ORF has a direct correlation with the accuracy of the prediction method, with large ORFs being the most accurate [one of the largest known prokaryotic ORFs identified to date is $s y r E, 28231$ nucleotides (51)]. At the opposite end of the size spectrum, minigenes (or peptides) are very difficult to predict and can be as small as two amino acids, $\lambda$ bar (57) and E. coli MR (129).

\section{Homology Search}

After predicting ORFs, the next phase is to apply a battery of algorithms 


\section{DRUG DISCOVERY AN D GEN OMIC TECHN OLOGIES}

designed to extract as much information about the ORF as possible. One of the most widely used methods to define function, and hence gene annotation, is to perform a search of existing sequence databases to determine if a sim ilar protein sequence has an associated function. The two main algorithms used for homology searching are basic local alignment search tool (BLAST) (3) and FASTA (99). BLAST uses a heuristic algorithm to detect relationships among sequences that share only isolated regions of similarity. The BLAST family of programs allows one to search protein or DNA databases regardless of whether the query sequence is amino acid or nucleic acid. FASTA is a heuristic algorithm that uses a modification of the optimization method described by Needleman and Wunsch (87) for global alignment and Smith and Waterman (118) for local alignment and may be more sensitive than
BLAST. Like BLAST, FASTA also allows one to search protein or DNA databases regardless of whether the query sequence is amino acid or nucleic acid. (For an overview of searching sequence databases, see Reference 89.) Two more advanced versions of the BLAST program that run faster due to requiring a "two-hit" method are Gapped BLAST and position-specific iterated BLAST (PSI-BLAST) (4). The new gapped alignment algorithm uses dynamic programming to extend a central pair of aligned residues in both directions. The PSI-BLAST program uses a position-specific score matrix (also called profiles or motifs) to detect weak but biologically relevant sequence similarities. Although this program may identify weak homologies to annotated entries in a database, it does require manual inspection of the output data between each iteration. Another search program for finding weak ho- mology is HMMER (http://hmmer. wustl.edu) (36). This allows one to use a multiple sequence alignment of known protein family members to build an HMM to search for new family members within a database or use a single protein sequence to search the Pfam HMM database as discussed below.

It is interesting that even today a significant fraction $(30 \%-50 \%$ of predicted ORFs) of both previously and newly sequenced genomes encode uncharacterized genes, including a significant number of strictly "orphan" genes, "ORFans" (ORFs with no detectable sequence similarity to proteins of other genomes) $(1,41)$.

\section{Sequence Databases}

At present, there are three major publicly available sequence database facilities (Table 1). The National Center for Biotechnology Information (NCBI) 


\section{DRUG DISCOVERY \\ AN D GEN OMIC TECH N OLOGIES}

Table 1. Listing of Various Bioinformatic Resources and Description

\begin{tabular}{|c|c|c|}
\hline \multicolumn{3}{|c|}{ Sequence Databases } \\
\hline $\mathrm{NCBI}$ & http://www.ncbi.nlm.nih.gov/ & $\begin{array}{l}\text { NCBI GenBank, PubMed, Entrez, and other databases } \\
\text { of published sequence data }\end{array}$ \\
\hline $\mathrm{EBI}$ & http://www.ebi.ac.uk/ & European Bioinformatics Institute \\
\hline DDBJ & http://www.ddbj.nig.ac.jp/ & DNA Data Bank of Japan \\
\hline PIR & http://www.mips.biochem.mpg.de/ & Protein Information Resource \\
\hline \multicolumn{3}{|c|}{ Domain or Pattern/Motif Databases and Tools } \\
\hline PROSITE & http://www.expasy.ch/prosite/ & $\begin{array}{l}\text { Database of protein families based on patterns and } \\
\text { weight matrices }\end{array}$ \\
\hline Blocks & http://blocks.fhcrc.org/ & $\begin{array}{l}\text { Multiply aligned, ungapped segments corresponding } \\
\text { to conserved regions of proteins }\end{array}$ \\
\hline Pfam & http://www.sanger.ac.uk/Software/Pfam/ & $\begin{array}{l}\text { Pfam-A contains a human-curated protein domain family. } \\
\text { Pfam-B contains automatically clustered families. }\end{array}$ \\
\hline ProDom & http://www.toulouse.inra.fr/prodom.html & $\begin{array}{l}\text { Automatically generated domains using a novel } \\
\text { procedure based on recursive PSI-BLAST searches }\end{array}$ \\
\hline PRINTS & http://www.bioinf.man.ac.uk/dbbrowser/PRINTS/ & $\begin{array}{l}\text { Database of protein fingerprints consisting of multiple } \\
\text { usually non-overlapping conserved motifs for a } \\
\text { protein family }\end{array}$ \\
\hline \multicolumn{3}{|c|}{ Subcellular Location } \\
\hline PSORT & http://psort.nibb.ac.jp/ & Expert system for protein localization \\
\hline ProtLock & Reference 21 & Statistical discrimination of amino acid content \\
\hline Chou and Elroo & Reference 24 & Discriminant function algorithm \\
\hline LocateProtein & Reference 113 & NN prediction \\
\hline \multicolumn{3}{|c|}{ Signal Sequence Determination } \\
\hline SignalP & http://www.cbs.dtu.dk/services/SignalP/ & NN and/or HMM signal sequence prediction \\
\hline Sigcleave & EMBOSS & Weight matrix \\
\hline SPScan & $\mathrm{GCG}$ & Weight matrix \\
\hline \multicolumn{3}{|c|}{ Transmembrane Prediction } \\
\hline DAS & http://www.sbc.su.se/ miklos/DAS/ & Dot-plot analysis against a protein dataset \\
\hline SOSUI & $\begin{array}{l}\text { http://sosui.proteome.bio.tuat.ac.jp/ } \\
\text { sosuiframe0E.html }\end{array}$ & $\begin{array}{l}\text { Average hydrophobicity with average amphiphilicity } \\
\text { index and positive-inside rule }\end{array}$ \\
\hline TopPred II & http://www.sbc.su.se/ erikw/toppred2/ & $\begin{array}{l}\text { Sliding window average of hydrophobicity and } \\
\text { positive-inside rule }\end{array}$ \\
\hline PHDhtm & http://cubic.bioc.columbia.edu/predictprotein/ & Trained NN and multiply aligned sequences \\
\hline TMHMM & http://www.cbs.dtu.dk/services/TMHMM/ & HMM trained on protein dataset \\
\hline PRED-TMR & http://o2.db.uoa.gr/PRED-TMR/ & Statistical analysis of SwissProt proteins \\
\hline $\begin{array}{l}\text { Lio and } \\
\text { Vannucci }\end{array}$ & Reference 74 & Wavelet change-point \\
\hline \multicolumn{3}{|l|}{ Software } \\
\hline EMBOSS & http://www.hgmp.mrc.ac.uk/Software/EMBOSS/ & Command line sequence analysis package \\
\hline GCG & http://www.accelrys.com/ & Command line sequence analysis package \\
\hline SeqWeb & http://www.accelrys.com/ & Web-based front end for GCG analyses \\
\hline Lasergene99 & http://www.dnastar.com/default.html & Windows sequence manipulation and analysis package \\
\hline MacVector & http://www.accelrys.com/ & Macintosh sequence manipulation and analysis package \\
\hline Vector NTI & http://www.informaxinc.com/ & Windows sequence manipulation and analysis package \\
\hline
\end{tabular}




\section{DRUG DISCOVERY \\ AN D GEN OMIC TECHNOLOGIES}

at the National Institutes of Health (NIH) was established in 1988 and has assumed responsibility for maintaining the sequence database GenBank ${ }^{\circledR}$ since 1992. GenBank consists of nucleotide and protein sequences including those from EBI and DDBJ (see below) as well as molecular modeling database (MMDB) and other sequence databases (12). The NCBI site contains other information such as clusters of orthologous groups (COGs) of proteins-phylogenetic classification), links to literature databases (such as PubMed), tools for data mining (such as BLAST and PSI-BLAST), access to Entrez (search and retrieval system), and much more.

The EMBL Nucleotide Sequence Database was established in 1980 at the European Molecular Biology Laboratory in Heidelberg, Germany. Since 1994, the EMBL Database has been maintained at the European Bioinformatics Institute (EBI), an EMBL outstation, located at the Wellcome Trust Genome Campus in Hinxton near Cambridge, UK. The EBI operates a number of databases, namely the EMBL Nucleotide Sequence Database including sequences from GenBank and DDBJ, translated protein sequences from EMBL (TrEMBL), SWISS-PROT (curated non-redundant protein sequences), Ensembl (automatic annotation on eukaryotic genomes), Macromolecular Structure Database (MSD), and many other databases (9). The EBI site contains additional information such as links to protein domain and motif database (InterPro) tools for data mining, database searching (SRS), homology searching (such as FASTA), analysis tools (such as ClustalW), and much more.

The DNA Data Bank of Japan (DDBJ) was established in 1986 and is located at the Center for Information $\mathrm{Bi}$ ology, National Institute of Genetics (NIG), Mishima, Japan (128). DDBJ is the sole DNA data bank in Japan and also contains sequences from GenBank and the EMBL Data Library. It provides similar tools for data retrieval (such as SRS), homology searching (such as PSI-BLAST, SSEARCH, and FASTA), analysis programs for multiple sequence alignment (such as ClustalW), secondary protein structure prediction (such as SSThread), and much more. All three data banks exchange data nightly and therefore contain the same data.

The Protein Information Resource (PIR), in collaboration with the Munich Information Center for Protein Sequences (MIPS) and the Japan International Protein Information Database (JIPID), produces the most comprehensive, well-annotated, and non-redundant protein sequence database in the public domain, called the PIR-International Proteins Sequence Database (Table 1) (10). A listing of other sequence and types of databases can be found in the annual database issue of Nucleic Acids Research (see Volume 29, Number 1, January 1, 2001).

Word of caution. With the fast pace of genomic sequences being deposited into the databases, there is also much pressure to provide annotation to the sequences. This comes with a risk of the data not being highly accurate $(45,119)$. Hence, the error rate of annotations for various genomes can be at least $8 \%$, as in the case for $M$. genitalium (18). Other genomes such as Chlamydia trachomatis and Methanococcus jannaschii have been reported to contain annotation errors in the database $(72,83)$. The probable main cause for errors is the sole reliance on automated computer methods to assign some type of a function based on similarity to other sequences that may contain annotation errors or using too low of a minimal comparison threshold value. Another major problem with the current automated annotation approaches is inconsistency (119). This puts pressure on the hand-curated databases (such as Amos Bairoch's SWISSPROT database at http://www.expasy. $\mathrm{ch} / \mathrm{sprot} / \mathrm{sprot} /$ ) to check the literature, reanalyze the sequence using other resources such as PROSITE or Pfam, and weed out those annotations without supportive information (68). The recent publication of the complete genomic sequence of Pseudomonas aeruginosa avoided the perpetuation of annotation errors by enlisting 61 researchers for the Pseudomonas Community Annotation Project to define ORFs and assign function (125). There is also a need to re-annotate completed genomes on an ongoing basis, as what was recently done for the genome of Mycoplasma pneumoniae (31), as newer algorithms and information become available.

\section{Domain or Pattern/Motif Databases and Tools}

As we have seen above, additional analysis other than just a BLAST search alone is needed for gene annotation. There exists a number of different domain/pattern or motif databases, each based on a slightly different philosophy with regard to sequence features that make up a subclass of proteins with known or presumed function (Table 1). PROSITE is a database of protein families and domains based on patterns and profiles/weight matrices (60). The profiles are constructed by various different techniques such as HMMs (38). Each protein family or domain listed in PROSITE comes with documentation providing information on the structure and function of these proteins. Blocks are multiply aligned, ungapped segments corresponding to the most conserved regions of proteins. The list of protein families used to construct the Blocks database were from Prosite, but, in the recent version, Blocks+, additional families are obtained from ProDom, Prints, Pfam, and DOMO (56). The user may search the Blocks database using a comparison BLIMPS search program or, as an alternative, the IMPLALA program, which searches PSI-BLAST-generated subsets of the Blocks database (56). Pfam is a database of protein domain families (11). Pfam-A contains curated multiple sequence alignments for each protein domain or conserved region generated from a non-redundant protein set of sequences from the SWISS-PROT and TrEMBL databases (68). With the latest 6.1 release of Pfam, supplemented domains, Pfam-B, have been constructed using the ProDom database. Profile HMMs have been built from the Pfam alignments and are a useful tool for finding these domains in a new sequence even if the homology is weak. ProDom is another database of protein domain families generated from the SWISS-PROT and TrEMBL sequence databases including the Pfam-A families (28). A set of complete genomes (archaea, bacteria, and eukaryote) is used to generate ProDom-CG. Both databases are available from the Web. PRINTS is a database of protein family "fingerprints" that occurred because of the limitations of single-motif 
pattern-matching methods that yield binary diagnoses with no biological context with which to assess the significance of the result (7). In the alignment of proteins within a family, more than one motif may exist. Hence, PRINTS yields information as to the number of fingerprint matches a new sequence has to a family, as well as researched information about the family and, where possible, links to known structural or functional data. The PRINTS database now includes a composite of SWISSPROT and TrEMBL and is called PRINTS-S (6). A listing of other types of domain and motif databases can be found in the annual database issue of Nucleic Acids Research (see Volume 29, Number 1, January 1, 2001).

There are a number of standalone tools that allow one to find motifs within a dataset. A software tool called TEIRESIAS is capable of detecting and reporting all existing patterns in an in- put set of sequences without pairwise alignments (107). TEIRESIAS scans the input sequences and locates elementary patterns that are then used in a convolution phase to construct the maximal pattern(s) in the dataset. The Gibbs motif sampling has been used to define motifs within immunoglobulin fold proteins, helix-turn-helix DNA binding proteins, and $\beta$-barrel strands in porins (88). Finally, a study was done to evaluate seven other different computational motif-finding methods (BLOCKMAK ER, ITERALIGN, MATCHBOX, MEME, PIMA, PROBE, and SAM) using a set of 20 reverse transcriptase protein sequences (63).

\section{Subcellular Location}

In bacterial genomes, $30 \%-50 \%$ of predicted ORFs are genes with no known associated function. In these cases when the standard search algo- rithms have failed to produce a function, methods based on amino acid composition alone may at least help to define the localization of the gene product (Table 1). One of the best known methods is an expert system called PSORT $(84,85)$ that uses various kinds of knowledge rules, based on 106 proteins with known subcellular locations, for predicting the location of proteins in bacteria. This program predicts the location of a protein in one of four subcellular regions, cytoplasm, inner mem brane, periplasm, or outer membrane, with an accuracy of $83 \%$. PSORT gives additional information such as determination of a signal sequence using McGeoch's method (80), cleavable signal sequence using von Heijne's method (136), lipoprotein based on a combination of McGeoch's method and the consensus motif by von Heijne (138), and transmembrane segments using ALOM software (69). A program called ProtLock does a statistical discrimination analysis of the amino acid content of a protein to predict the cellular location of the protein (21). ProtLock defines five categories of subcellular location (integral membrane, anchored membrane, extracellular, intracellular, and nuclear) that are used for identifying proteins with about $76 \%$ accuracy based on a total training set of 1000 proteins. Using correlation analysis of the amino acid composition and cellular location of a protein, Reinhardt and Hubbard (105) have developed a program to define the three subcellular locations in prokaryotic organisms (cytoplasmic, extracellular, and periplasmic) based on neural networks (NNs) trained on sequences whose subcellular location was annotated. The reported accuracy of Reinhardt and Hubbard's (105) NN was $81 \%$ based on jackknife analysis using a dataset of 993 proteins extracted from the SWISS-PROT database. A discriminant function algorithm developed by Chou and Elrod (24) gave an accuracy prediction range of $87 \%-91 \%$ for the same set of three subcellular locations. Chou and Elrod (24) used the dataset produced by Reinhardt and Hubbard (105) (with an additional four proteins) for training and evaluation; however, their method of performing the jackknife test was slightly different than Reinhardt and Hubbard. Using an 
improved neural network implementation, Schneider (113) developed a program called LocateProtein with a crossvalidated average prediction accuracy of $93 \%$ based on the same dataset as Chou and Elrod (24). LocateProtein only seeks to classify a protein as either cytoplasmic or non-cytoplasmic.

\section{Signal Sequence Determination}

Most of the bacterial proteins that are translocated to the cell membrane contain a signal sequence (also called signal peptide or SP) at the N-terminal region of the protein. This sequence consists of three distinct regions: an $\mathrm{N}$ terminal positively charged n-region, a hydrophobic h-region, and a neutral polar c-region (92). For cleavage, the residues at positions -3 and -1 must be small and neutral (137). A program called SignalP uses a combined NN approach to predict the recognition of SPs and their cleavage sites (92). A more recent HMM version of SignalP has been developed and is also available on the Web (90). The performance values of SignalP between the NN and HMM versions show the NN method to be slightly better at predicting the correct bacterial cleavage site, while the HMM method gives minor improvement in discriminating true SP from non-secretory peptide sequences. Another program for predicting SPs is Sigcleave program that uses the weight matrix method of von Heijne (136). SPScan from Genetics Computer Group (Madison, WI, USA) is similar to Sigcleave but also uses the weight matrices given in Nielsen et al. (91) in concert with McGeoch's description of a minimum acceptable signal peptide (80). Since these programs are based on different prediction methods, it may be wise to use multiple programs in concert. There are also various programs available for predicting $\mathrm{N}$-terminal sorting signals (see Table 1 and Reference 25). Recently, Chou (23) has shown that the discriminate function techniques applied to subcellular localization of proteins can also be used to identify signal sequences and predict their cleavage sites. The discriminate function algorithm detects common features of signal sequences without regard for differing lengths, sequences, and composition.

\section{Transmembrane Segments}

One of the main differences between Gram-negative and Gram-positive bacteria is the cell wall structure. Gramnegative bacteria have two lipid bilayers - an inner membrane layer and an outer membrane layer. Between these two membrane layers lies the periplasmic space that contains the peptidoglycan matrix. Gram-positive bacteria have only a single membrane layer that is surrounded on the outside by the peptidoglycan matrix.

\section{Inner Membrane Proteins}

Typically in the inner membrane of Gram-negative bacteria and in the single membrane of Gram-positive bacteria, integral membrane proteins are held in place by hydrophobic interactions with the lipid bilayer. Aside from lipoproteins that are anchored to the membrane via a $\mathrm{N}$-terminal lipid moiety, membrane proteins consist of at least one strongly predicted $\alpha$-helical transmembrane segment (67). These segments typically consist of a stretch of hydrophobic amino acids, 18-24 residues long. One of the early prediction methods for being able to distinguish transmembrane segments from hydrophobic segments of soluble proteins used a sliding window (39). This method allowed both the residue hydrophobicity value and the $\alpha$-helix hydrophobic moments to be computed and displayed in a hydrophobic moment plot of the entire protein. Exam ples of transmembrane prediction methods currently available over the Web are the discriminant analysis method (69) employed in the PSORT program mentioned above, the dense alignment surface (DAS) method (29), and the system SOSUI (Table 1) (59). The accuracy in predicting transmem brane helices in the correct location can be approximately 95\% (120). However, predicting the correct topology of the transmembrane segments within a protein (i.e., how it transverses the membrane, inward or outward) is at best about $75 \%$ accurate for a single sequence-based hydrophobicity/discriminant analysis and as high as $86 \%$ accurate using multiple protein alignments. The amino acid composition and the 
observation that there is a greater abundance of positively charged amino acids in the cytoplasmic segment versus the periplasmic segment of integral membrane proteins ("positive-inside" rule) play an important role in determining the topology $(67,86,135)$. There are various programs that predict the transmembrane helices and topology in a protein. TopPred II (26) incorporates the positive-inside rule and compiles other existing knowledge about the topology. PHDhtm performs a local alignment to generate a profile of a protein and then two levels of neural network systems along with a refinement of topology using the positive residue content of the non-transmembrane loop regions to predict transmembrane segments (108). TMHMM (120) uses an HMM for predicting transmembrane segments and has recently been tested on complete genomes predicting that $20 \%-30 \%$ of all genes encode mem brane proteins (70). PRED-TMR uses statistical analysis of transmembrane proteins in the SwissProt database for defining potential termini of transmem brane regions (97). Nilsson et al. (94) concluded that by using the consensus of five different membrane protein topology prediction methods, a high level of topology prediction can be achieved by a simple majority vote approach. A recent paper by Lio and Vannucci (74) uses a non-parametric method based on a wavelet changepoint analysis and new propensity scale to predict transmembrane segments. A comparison of this wavelet changepoint analysis system with a number of other transmembrane prediction programs was demonstrated in the paper. Both the source code and database are available upon request of the authors.

\section{Outer Membrane Proteins}

The outer membrane proteins (OMPs) form a distinct class of integral membrane proteins (for overview of OMPs, see References 19 and 122). Porins are a major class of OMPs. These proteins typically contain regions of amphipathic $\beta$-pleated sheet structure (alternating hydrophobic/hydrophilic amino acids) that transverse the mem brane 8-22 times and form $\beta$-barrel structures. Schirmer and Cowan (112) used a modified $\beta$-side-hydrophobicity and a sliding window width of four residues for every second residue to correctly predict 16 out of the $18 \beta$-strands of LamB. Neuwald et al. (88) used a new Gibbs sampling algorithm to detect motif-encoding regions or patterns. When applied to a set of 32 bacterial OMPs, the sampler detected repeats that corresponded to $\beta$-strands (34). Gromiha et al. (50) used a rule-based approach for identifying transmembrane $\beta$ strands for a limited set of three different $16 \beta$-strands porins. A neural network algorithm was trained on a set of seven atomically determined porins to predict outer membrane $\beta$-strands (34). This program can be accessed at: http://loop8.biologie.uni-konstanz.de/ kay/om_topo_predict.html. Topology analysis of OmpA predicted at least 16 transmembrane $\beta$-strands. However, high-resolution structure analysis of OmpA reveals only eight transmem brane $\beta$-strands (98). Recently, Gnanasekaran et al. (47) have developed a method using structurally conserved profiles from structurally conserved porin regions to identify or discriminate $\beta$-strands. However, it is recommended that other algorithms be used in combination with this method for searching other protein databases. In the genomic analysis of Helicobacter pylori, strain 26695, a family of 32 putative OMPs was identified based on the "characteristic alternating hydrophobic residues at their carboxy termini" (132). Four of these OMPs were identified as porins based on identity to published sequences. Unfortunately, all of these methods have shown only limited success in defining transmembrane $\beta$ strands, and better methods are needed.

\section{Short Nucleotide Repeat Regions}

Prokaryotic genomes contain regions of repetitive DNA in the form of homopolymeric tracts [i.e., poly(dA), poly(dG), etc.] and homogeneous or heterogeneous tracts. For a review on short DNA repeat sequences in prokaryotic genomes, see van Belkum et al. (133). Gain or loss of the repeat regions through slipped-strand mispairing is one mechanism of genotypic variation (82). Since the publication of the first bacterial genome, many types of short repeat sequences have been identified in pathogenic organisms. These repeats localize within the coding or promoter region of genes involved in virulence. One of the mechanisms that bacteria have in trying to evade the host immune system is to change its appearance by either phase variation (the turning "on" or "off" of a gene) or antigenic variation (modification of the gene production). In the genomic sequence of $N$. meningitidis serogroup A strain Z2491, there are about 26 tandem repeats ranging from homopolymeric tracts to di-, tetra-, and pentanucleotide repeats found mostly within the coding sequences. The repeats were found mainly in surface-exposed genes or genes involved in the biosynthesis or modification of surface structures (96). In another strain of $N$. meningitidis, serogroup B strain MC58, 65 potential phase variable genes were identified, and, of these, only 13 were previously recognized (110). This shows the fruitfulness of genomic analysis for the identification of novel genes presumed to be responsible for immunogenic variation. Repeat sequences have also been found in other human pathogens such as Haemophilus influenzae and $H$. pylori $(62,111)$.

The ability to identify these potential immunogenic variable genes is im portant in the decision of whether the gene(s) might be a good vaccine candidate. Certainly, if the gene product were not expressed or present during pathogenesis, it most likely would not be considered a worthwhile vaccine target. A DNA tandem repeat finder program has been developed by Benson (13) and is available over the Web at http://c3.biomath.mssm.edu/trf.html.

\section{Software and Computers}

There are a number of commercially available programs that can perform many of the analysis functions described above (Table 1). Probably the most widely used and known UNIX ${ }^{\circledR}$ software is the Wisconsin Package ${ }^{\circledR}$ (Accelrys, San Diego, CA, USA). Seq$\mathrm{Web}^{\circledR}$ is a Web interface of the GCG software. Both of these software packages are also available from Accelrys. Other desktop operating sequence analysis software are Lasergene99 
(Microsoft $^{\circledR}$ Windows $^{\mathrm{TM}}$ or Macintosh $^{\circledR}$ ) (DNASTAR, Madison, WI, USA); MacVector ${ }^{\circledR}$ (Macintosh) and OMIGA (Windows), both from GCG or Oxford Molecular Group; and Vector NTI ${ }^{\circledR}$ Suite (Windows) from InforMax (North Bethesda, MD, USA). The European Molecular Biology Open Software Suite (EMBOSS) is an extensive, free, open-source UNIX software analysis package developed for the needs of the molecular biology community. Most research facilities customize the software needed for data analysis and how the output data is presented based on their specific needs. This process is constantly evolving as new software and methods are being developed.

Dealing with the vast quantity of data produced through computational genomics provides a relatively daunting task in itself. Many different efforts exist to help standardize the input and output formats that various new analysis methods handle or provide. In general, how ever, the standards that are most widely used are de facto standards that have grown out of a need for simplicity.

The quantity of data generated in analyzing an entire genome can be enormous. For a relatively small bacterial genome like $C$. trachomatis, about 1 Mbp, as much as $500 \mathrm{MB}$ data can easily be generated in the process of storing all of the different analysis results for the ORFs. While that quantity of data is not terribly significant given the size of today's mass storage devices, managing that quantity of data to allow easy storage and retrieval requires some thought and planning. In any significant genomic mining program, time and effort must be put into developing a scheme for storing the data on top of the mechanism for generating that data.

As with every field related directly to computers, all of the major hardware platforms are represented in one way or another with different available software (MacVector runs on Macintosh, Lasergene 99 runs under Windows, and GCG and EMBOSS run under most flavors of UNIX). Many of the tools described in earlier sections have been developed in $\mathrm{C}$ or Perl and could potentially be ported to any platform desired. In the sim plest case, a new computational genomics laboratory would do well with a UNIX machine with 512 MB RAM and
32 GB disk space. This would provide the computational power and data storage capabilities necessary to begin analyzing whole bacterial genomes.

\section{PUTTING IT ALL TOGETHER}

A nice example of using genomics for bacterial vaccine development is the group from Chiron S.p.A. (Siena, Italy), who, in collaboration with TIGR, mined the genome of $N$. meningitidis, serogroup B $(\mathrm{NmB})$ to identify possible vaccine candidates $(101,130)$. The approach Pizza et al. (101) took was to search the genome for potentially encoded novel surface-exposed or exported proteins in the development of a protein-based vaccine. The first step was to determine the complete genomic sequence of a virulent strain of $\mathrm{NmB}$ and prediction of ORFs (130). ORFs that were determined to code for cytoplasmic proteins were not investigated further. All other ORFs were further analyzed to identify proteins that were exposed to the outside of the cell using various programs such as PSI-BLAST, FASTA, MOTIFS, FINDPATTERNS, and PSORT. Databases such as ProDom, Pfam, and Blocks were used to predict surface-associated proteins such as transmembrane domains, leader peptides, homologies to known surface proteins, lipoprotein signature, outer mem brane anchoring motives, and host cell binding domains such as the tripeptide, arginine-glycine-aspartic acid (RGD) (66). Out of a total of 2158 predicted ORFs, 570 were predicted to be surface exposed or exported. These ORFs were cloned and expressed as fusion proteins in E. coli. Three hundred fifty of the proteins were successfully expressed, purified, and used to immunize mice. The sera obtained were tested in a whole cell ELISA, fluorescence-activated cell sorter (FACS) system, and for bactericidal activity (a property known to correlate with vaccine efficacy in humans) to verify cell surface presence of the proteins. Eighty-five proteins were found to be strongly positive in at least one of the assays above. Of these, seven representative proteins that were positive in all three assays and whose genes were not predicted to be phase variable were selected for further study. Surprisingly, these seven surface exposed proteins are conserved among all $31 \mathrm{~N}$. meningitidis strains tested. Presumably, any or all of these seven vaccine ORF candidates would be moved into the next pre-clinical phase of vaccine development.

In a recent report, Wizemann et al. (140) used similar genomic algorithms, as mentioned above, for the prediction of surface-localized proteins from the genomic sequence of $S$. pneumoniae. A major difference in their approach than what was done with $\mathrm{NmB}$ was to test their vaccine candidates directly in an animal model to screen the proteins for vaccine efficacy. In this way, they were able to identify two vaccine candidates that conferred protection in mice but were not detected on laboratory-grown bacteria by FACS analysis. Either the amount or accessibility of these antigens on the bacteria is limited or these proteins represent a novel class of proteins that are only expressed during in vivo infection.

In another example, Gomez et al. (48) searched the genome of Mycobacterium tuberculosis for potential secreted proteins. They used various mining tools such as SignalP, SPScan, TMpred, and lipoprotein PrositeScan to identify various classes of secreted proteins. A subclass of the predicted secreted proteins was confirmed in the laboratory by constructing $p h o A^{\prime}$ fusions.

These few examples highlight current approaches whereby genomic analysis and screening is done in silico before any wet laboratory research. Although we have become smarter, faster, and better at predicting vaccine candidates, the need and the time required to do experimental laboratory and clinical studies still remains unchanged in the development of safe and efficacious vaccines.

\section{WHAT NEXT?}

So where do we go from here? Much work is still needed, as even with the best algorithms available today, proteins are being found on the cell surface of the bacterium that were not predicted to be surface exposed. Examples of such findings are the ribosomal protein L12 of Neisseria gonorrhoeae as being membrane associated and surface exposed (121); a histone-like protein 
and $\mathrm{S} 4$ and $\mathrm{S} 5$ ribosomal proteins were isolated from the cell wall of Mycobacterium leprae (79); and, in the analysis of OMPs of $H$. pylori, translational elongation factor EF-Tu, urease $\alpha$ and $\beta$ subunits, catalase and others were found (93). More and more examples of this type will be forthcoming as we continue the analysis of bacterial surface proteins. What does this mean? The answer is simple: researchers should not limit their analysis to just genomic mining.

Transcription profiling using oligonucleotide or DNA microarrays is a technology geared for high-throughput genomic mRNA expression analysis. This method uses genomic gene predictions for the design of the array and subsequent hybridization of the mRNA, either directly or indirectly (cDNA), to the array for detection and has shed tremendous light on the gene expression profile of the entire bacterial genome grown under various conditions $(5,106)$ or as a means to monitor bacteria-host cell interactions $(27,30)$. However, there are some technical difficulties using this method for identifying vaccine candidates: $(i)$ the level of mRNA transcripts may not correlate with the actual amount of protein expressed $(5,53)$; ( $i i)$ the stability of most bacterial mRNAs are in the order of minutes (104); and (iii) patterns of gene expression are not the ideal method for discovery of vaccine targets (20).

An area of concern using just genomics and/or transcriptional analysis for protein expression is the difficulty in defining post-transcriptional modifications of the expressed protein and in predicting genes due to frameshifts or alternative RNA splice sites (117), hence the need to identify all the proteins made by the genome, termed proteome, through proteomics. Proteomics is now at the level of sensitivity and high throughput to be able to complement genomics and give a more realistic analysis of the actual proteins expressed by the organism. In a search for potential vaccine candidates in $H$. pylori, Nilsson et al. (93) used a combination of liquid-phase isoelectric focusing and gel separation for the isolation of $H$. pylori proteins. These proteins were then digested with trypsin and analyzed on a matrix-assisted laser desorption/ioniza- tion time-of-flight (MALDI-TOF) mass spectrometer with subsequent database searching for the identification of the protein. Chakravarti et al. (22) also showed how the combined use of genomics and proteomics can help identify potential vaccine candidates for $H$. influenzae as well as $H$. pylori. For a recent general review on how vaccinology will benefit from genomic and proteom ic technologies, see Grandi (49).

Knowing that no one method alone can identify all of the proteins of interest, researchers are combining all three disciplines of genomics, transcription profiling, and proteomics (142). Whether this will be the future for vaccine discovery programs remains to be seen. The next key level of importance for bacterial vaccine discovery is to be able to determine what proteins are made during infection, in vivo, as it is already known that type III effector proteins and virulence factors are only expressed during eukaryotic cell contact (64). The questions now being asked are: "Are we missing good vaccine candidates by focusing our study of laboratory-grown bacteria?" and "How do we identify these vaccine targets induced in vivo?"

\section{NOW AND BEYOND}

The recent release of the draft sequence of the human genome $(73,134)$ has opened the door for newer methods to be developed both in the analysis and detection of genes and their proteins. Many of these methods can and will be applied to the discovery of new pharmaceutical targets and hopefully will lead to the development of new treatments. On the bacterial vaccine discovery side, better approaches are needed to provide insight into pathogenesis that may lead to new interventions to prevent and treat infection (126). How ever, until that time, as more and more genomic sequences are being completed, improved laboratory methods and algorithms are needed to help sort and understand all of the present data, especially the substantial proportion of ORFs that have no known function (139). In this latter case, new computational approaches and genomic context are being developed to help define pu- tative gene function $(46,141)$. Comparisons between genomes, especially of the same genus, may help to define vaccine candidates (123). In a way, this marks the end of the early genome mining era and the start of a relatively new area of scientific discipline called functional genomics $(35,46)$.

\section{ACKNOWLEDGMENTS}

The authors wish to thank Deb Chakravarti, Leah Fletcher, George Maalouf, John McMichael, Stephen Olmsted, Burkhard Rost, Steven Salzberg, Guenter Stoesser, Yoshio Tateno, George Weinstock, and Rose Marie Woodsmall for providing valuable editorial comments on various sections of the manuscript. We also appreciate the help of Susan Hoiseth and Laura York with part of the Introduction, as well as Terri Ward and Eileen Shirley for their endless librarian help.

\section{REFERENCES}

1.Alimi, J., O. Poirot, F. Lopez, and J. Claverie. 2000. Reverse transcriptase-polymerase chain reaction validation of 25 "orphan" genes from Escherichia coli K-12 MG1655. Genome Res. 10:959-966.

2.Alm, R.A., L.S. Ling, D.T. Moir, B.L. King, E.D. Brown, P.C. Doig, D.R. Smith, B. Noonan et al. 1999. Genomic-sequence comparison of two unrelated isolates of the human gastric pathogen Helicobacter pylori. Nature 397:176-180.

3.Altschul, S.F., W. Gish, W. Miller, E.W. Myers, and D.J. Lipman. 1990. Basic local alignment search tool. J. Mol. Biol. 215:403410.

4.Altschul, S.F., T.L. Madden, A.A. Schaffer, J. Zhang, Z. Zhang, W. Miller, and D.J. Lipman. 1997. Gapped BLAST and PSIBLAST: a new generation of protein database search programs. Nucleic Acids Res. 25:3389-3402.

5.Ang, S., C.Z. Lee, K. Peck, M. Sindici, U. Matrubutham, M.A. Gleeson, and J.T. Wang. 1679. Acid-induced gene expression in Helicobacter pylori: study in genomic scale by microarray. Infect. Immun. 69:1679-1686.

6.Attwood, T.K., M.D. Croning, D.R. Flower, A.P. Lewis, J.E. Mabey, P. Scordis, J.N. Selley, and W. Wright. 2000. PRINTSS: the database formerly known as PRINTS. Nucleic Acids Res. 28:225-227.

7.Attwood, T.K., D.R. Flower, A.P. Lewis, J.E. Mabey, S.R. Morgan, P. Scordis, J.N. Selley, and W. Wright. 1999. PRINTS prepares for the new millennium. Nucleic Acids Res. 27:220-225. 
8.Audic, S. and J.M. Claverie. 1998. Selfidentification of protein-coding regions in microbial genomes. Proc. Natl. Acad. Sci. USA 95:10026-10031.

9.Baker, W., A. van den Broek, E. Camon, P. Hingamp, P. Sterk, G. Stoesser, and M.A. Tuli. 2000. The EMBL nucleotide sequence database. Nucleic Acids Res. 28:19-23.

10.Barker, W.C., J.S. Garavelli, Z.L. Hou, H.Z. Huang, R.S. Ledley, P.B. McGarvey, H.W. Mewes, B.C. Orcutt et al. 2001. Protein Information Resource: a community resource for expert annotation of protein data. Nucleic Acids Res. 29:29-32.

11.Bateman, A., E. Birney, R. Durbin, S.R. Eddy, K.L. Howe, and E.L. Sonnhammer. 2000. The Pfam protein families database. Nucleic Acids Res. 28:263-266.

12.Benson, D.A., I. Karsch-Mizrachi, D.J. Lipman, J. Ostell, B.A. Rapp, and D.L. Wheeler. 2000. GenBank. Nucleic Acids Res. 28:15-18.

13.Benson, G. 1999. Tandem repeats finder: a program to analyze DNA sequences. Nucleic Acids Res. 27:573-580.

14.Benton, D. 1996. Bioinformatics-principles and potential of a new multidisciplinary tool. Trends Biotechnol. 14:261-272.

15.Black, S., H. Shinefield, B. Fireman, E. Lewis, P. Ray, J.R. Hansen, L. Elvin, K.M. Ensor et al. 2000. Efficacy, safety and im munogenicity of heptavalent pneumococcal conjugate vaccine in children. Northern California Kaiser Permanente Vaccine Study Center Group. Ped. Infect. Dis. J. 19:187195

16.Blattner, F.R., G. Plunkett, III, C.A. Bloch, N.T. Perna, V. Burland, M. Riley, J. Collado-Vides, J.D. Glasner et al. 1997. The complete genome sequence of Escherichia coli K-12. Science 277:1453-1474.

17.Borodovsky, M. and J. McIninch. 1993. Prediction of gene locations using DNA Markov chain models, p. 231-248. In H.A. Lim, J.W. Fickett, C.R. Cantor, and R.J. Robbins (Eds.), The Second International Conference on Bioinformatics, Supercomputing, and Complex Genome Analysis. World Scientific Publishing, River Edge, N.J.

18.Brenner, S.E. 1999. Errors in genome annotation. Trends Genet. 15:132-133.

19.Buchanan, S.K. 1999. Beta-barrel proteins from bacterial outer membranes: structure, function and refolding. Curr. Opin. Struct. Biol. 9:455-461.

20.Carucci, D. 2001. Genomic tools for gene and protein discovery in malaria: toward new vaccines. Vaccine 19:2315-2318.

21.Cedano, J., P. Aloy, J.A. Perez-Pons, and E. Querol. 1997. Relation between amino acid composition and cellular location of proteins. J. Mol. Biol. 266:594-600.

22.Chakravarti, D., M. Fiske, L. Fletcher, and R. Zagursky. 2000. Application of genomics and proteomics for identification of bacterial gene products as potential vaccine candidates. Vaccine 19:601-612

23.Chou, K.-C. 2001. Prediction of protein signal sequences and their cleavage sites. Proteins Struct. Funct. Genet. 42:136-139.

24.Chou, K.C. and D.W. Elrod. 1998. Using discriminant function for prediction of sub- cellular location of prokaryotic proteins. Biochem. Biophys. Res. Commun. 252:6368

25.Claros, M.G., S. Brunak, and G. von Heijne. 1997. Prediction of N-terminal protein sorting signals. Curr. Opin. Struct. Biol. 7:394-398

26.Claros, M.G. and G. von Heijne. 1994 TopPred II: an improved software for mem brane protein structure predictions. Comput. Appl. Biosci. 10:685-686.

27.Coombes, B.K. and J.B. Mahony. 2001. cDNA array analysis of altered gene expression in human endothelial cells in response to Chlamydia pneumoniae infection. Infect. Immun. 69:1420-1427.

28.Corpet, F., F. Servant, J. Gouzy, and D. Kahn. 2000. ProDom and ProDom-CG: tools for protein domain analysis and whole genome comparisons. Nucleic Acids Res. 28:267-269.

29.Cserzo, M., E. Wallin, I. Simon, G. von Heijne, and A. Elofsson. 1997. Prediction of transmembrane $\alpha$-helices in prokaryotic membrane proteins: the dense alignment surface method. Protein Eng. 10:673-676.

30.Cummings, C.A. and D.A. Relman. 2000 Using DNA microarrays to study host-microbe interactions. Emerg. Infect. Dis. 6:513525.

31.Dandekar, T., M. Huynen, J.T. Ragula, B. Ueberle, C.U. Zimmermann, M.A. Andrade, T. Doerks, L. Sanchez-Pulido et al. 2000. Re-annotating the Mycoplasma pneumoniae genome sequence: adding value, function and reading frames. Nucleic Acids Res. 28:3278-3288.

32.DasSarma, S., U.L. RajBhandary, and H.G. Khorana. 1984. Bacterio-opsin mRNA in wild-type and bacterio-opsin deficient Halobacterium halobiumstrains. Proc. Natl. Acad. Sci. USA 81:125-130.

33.Delcher, A.L., D. Harmon, S. Kasif, O. White, and S.L. Salzberg. 1999. Improved microbial gene identification with GLIMMER. Nucleic Acids Res. 27:4636-4641.

34.Diederichs, K., J. Freigang, S. Umhau, K. Zeth, and J. Breed. 1998. Prediction by a neural network of outer membrane $\beta$-strand protein topology. Protein Sci. 7:2413-2420.

35.Dunham, I. 2000. Genomics-the new rock and roll? Trends Genet. 16:456-461.

36.Durbin, R., S.R. Eddy, A. Krogh, and G. Mitchison. 1998. Biological Sequence Analysis: Robabilistic Models of Proteins and Nucleic Acids. Cambridge University Press, Cambridge.

37.Eby, R. 1995. Pneumococcal conjugate vaccines, p. 695-718. In M.F. Powell and M.J. Newman (Eds.), Pharmceutical Biotechnology, vol. 6. Plenum Press, New York.

38.Eddy, S.R. 1996. Hidden Markov models. Curr. Opin. Struct. Biol. 6:361-365.

39.Eisenberg, D., E. Schwarz, M. Komaromy, and R. Wall. 1984. Analysis of membrane and surface protein sequences with the hydrophobic moment plot. J. Mol. Biol. 179:125-142.

40.Fedson, D.S., D.M. Musher, and J. Eskola. 1988. Pneumococcal vaccine, p. 553-607. In S.A. Plotkin and W.A. Orenstein (Eds.), Vaccines, 3rd ed. Saunders Co., Philadelphia.
41.Fischer, D. and D. Eisenberg. 1999. Finding families for genomic ORFans. Bioinformatics 15:759-762.

42.Fleischmann, R.D., M.D. Adams, O. White, R.A. Clayton, E.F. Kirkness, A.R Kerlavage, C.J. Bult, J.F. Tomb et al. 1995 Whole-genome random sequencing and assembly of Haemophilus influenzae Rd. Science 269:496-512.

43.Frishman, D., A. Mironov, and M. Gelfand. 1999. Starts of bacterial genes: estimating the reliability of computer predictions. Gene 234:257-265.

44.Frishman, D., A. Mironov, H.W. Mewes, and M. Gelfand. 1998. Combining diverse evidence for gene recognition in completely sequenced bacterial genomes. Nucleic Acids Res. 26:2941-2947.

45.Galperin, M.Y. and E.V. Koonin. 1999 Functional genomics and enzyme evolution. Homologous and analogous enzymes encoded in microbial genomes. Genetica 106:159170.

46.Galperin, M.Y. and E.V. Koonin. 2000 Who's your neighbor? New computational approaches for functional genomics. Nat. Biotechnol. 18:609-613.

47.Gnanasekaran, T., S. Peri, A. Arockiasamy, and S. Krishnaswamy. 2000. Profiles from structure based sequence alignment of porins can identify $\beta$ stranded integral membrane proteins. Bioinformatics 16:839-842.

48.Gomez, M., S. Johnson, and M.L. Gennaro. 2000. Identification of secreted proteins of Mycobacterium tuberculosis by a bioinformatic approach. Infect. Immun. 68:2323-2327

49.Grandi, G. 2001. Antibacterial vaccine design using genomics and proteomics. Trends Biotechnol. 19:181-188

50.Gromiha, M.M., R. Majumdar, and P.K. Ponnuswamy. 1997. Identification of mem brane spanning $\beta$ strands in bacterial porins. Protein Eng. 10:497-500.

51.Guenzi, E., G. Galli, I. Grgurina, D.C. Gross, and G. Grandi. 1998. Characterization of the syringomycin synthetase gene cluster. A link between prokaryotic and eukaryotic peptide synthetases. J. Biol. Chem. 273:32857-32863.

52.Gussin, G.A., A.D. Johnson, C.O. Pabo, and R.J. Sauer. 1983. Repressor and cro protein: structure, function, and role in lysogenization, p. 93-121. In R.W. Hendrix, J.W. Roberts, F.W. Stahl, and R.A. Weisberg (Eds.), Lambda II. CSH Laboratory Press, Cold Spring Harbor, NY

53.Gygi, S.P., Y. Rochon, B.R. Franza, and R. Aebersold. 1999. Correlation between protein and mRNA abundance in yeast. Mol. Cell. Biol. 19:1720-1730.

54.Hannenhalli, S.S., W.S. Hayes, A.G. Hatzigeorgiou, and J.W. Fickett. 1999. Bacteria start site prediction. Nucleic Acids Res. 27:3577-3582.

55.Hanych, B., S. Kedzierska, B. Walderich, B. Uznanski, and A. Taylor. 1993. Expression of the Rz gene and the overlapping Rz1 reading frame present at the right end of the bacteriophage lambda genome. Gene 129:1 8. 
56.Henikoff, J.G., E.A. Greene, S. Pietrokovski, and S. Henikoff. 2000. Increased coverage of protein families with the blocks database servers. Nucleic Acids Res. 28:228-230

57.Hernandez-Sanchez, J., J.G. Valadez, J.V. Herrera, C. Ontiveros, and G. Guarneros. 1998. lambda bar minigene-mediated inhibition of protein synthesis involves accumulation of peptidyl-tRNA and starvation for tRNA. EMBO J. 17:3758-3765.

58.Herr, A.J., J.F. Atkins, and R.R. Gesteland. 2000. Coupling of open reading frames by translational bypassing. Annu. Rev. Biochem. 69:343-372.

59.Hirokawa, T., S. Boon-Chieng, and S. Mitaku. 1998. SOSUI: classification and secondary structure prediction system for mem brane proteins. Bioinformatics 14:378-379.

60.Hofmann, K., P. Bucher, L. Falquet, and A. Bairoch. 1999. The PROSITE database, its status in 1999. Nucleic Acids Res. 27:215-219.

61.Hoiseth, S. 2000. Vaccines, Bacterial, p. 767-778. In J. Lederberg (Ed.), Encyclopedia of Microbiology, Second ed., vol. 4. Academic Press.

62.Hood, D.W., M.E. Deadman, M.P. Jennings, M. Bisercic, R.D. Fleischmann, J.C. Venter, and E.R. Moxon. 1996. DNA repeats identify novel virulence genes in Haemophilus influenzae. Proc. Natl. Acad. Sci. USA 93:11121-11125.

63.Hudak, J. and M.A. McClure. 1999. A comparative analysis of computational motif-detection methods, p. 138-149. In R.B. Altman, A.K. Dunker, L. Hunter, T.E. Klein and K. Lauderdale (Eds.), Pacific Synmposium on Biocomputing '99, World Scientific, Singapore.

64.Hueck, C.J. 1998. Type III protein secretion systems in bacterial pathogens of animals and plants. Microbiol. Mol. Biol. Rev. 62:379-433.

65.Inamine, J.M., K.C. Ho, S. Loechel, and P.C. Hu. 1990. Evidence that UGA is read as a tryptophan codon rather than as a stop codon by Mycoplasma pneumoniae, Mycoplasma genitalium and Mycoplasma gallisepticum J. Bacteriol. 172:504-506.

66.Isberg, R.R. and G. Tran Van Nhieu. 1994. Binding and internalization of microorganisms by integrin receptors. Trends Microbiol. 2:10-14.

67.Jones, D.T., W.R. Taylor, and J.M. Thornton. 1994. A model recognition approach to the prediction of all-helical membrane protein structure and topology. Biochemistry 33:3038-3049.

68.Junker, V., S. Contrino, W. Fleischmann, H. Hermjakob, F. Lang, M. Magrane, M.J. Martin, N. Mitaritonna, C. O'Donovan, and R. Apweiler. 2000. The role SWISS-PROT and TrEMBL play in the genome research environment. J. Biotechnol. 78:221-234.

69.Klein, P., M. Kanehisa, and C. DeLisi. 1985. The detection and classification of membrane-spanning proteins. Biochim. Biophys. Acta. 815:468-476.

70.Krogh, A., B. Larsson, G. von Heijne, and E. Sonnhammer. 2001. Predicting trans- membrane protein topology with a hidden Markov model: application to complete genomes. J. Mol. Biol. 305:567-580.

71.Kyrpides, N.C. 1999. Genomes OnLine Database (GOLD 1.0): a monitor of com plete and ongoing genome projects worldwide. Bioinformatics 15:773-774.

72.Kyrpides, N.C. and C.A. Ouzounis. 1999. Whole-genome sequence annotation: "going wrong with confidence". Mol. Microbiol. 32:886-887.

73.Lander, E.S., L.M. Linton, B. Birren, C. Nusbaum, M.C. Zody, J. Baldwin, K. Devon, K. Dewar et al. 2001. Initial sequencing and analysis of the human genome. Nature 409:860-921.

74.Lio, P. and M. Vannucci. 2000. Wavelet change-point prediction of transmembrane proteins. Bioinformatics 16:376-382.

75.Liu, Z., M. Reches, I. Groisman, and H. Engelberg-Kulka. 1998. The nature of the minimal "selenocysteine insertion sequence" (SECIS) in Escherichia coli. Nucleic Acids Res. 26:896-902.

76.Loessner, M.J., S. Gaeng, and S. Scherer. 1999. Evidence for a holin-like protein gene fully embedded out of frame in the endolysin gene of Staphylococcus aureus bacteriophage 187. J. Bacteriol. 181:4452-4460.

77.Loh, J., M.G. Stacey, M.J. Sadowsky, and G. Stacey. 1999. The Bradyrhizobium japonicum nolA gene encodes three functionally distinct proteins. J. Bacteriol. 181:1544-1554.

78.Lukashin, A.V. and M. Borodovsky. 1998. GeneMark.hmm: new solutions for gene finding. Nucleic Acids Res. 26:1107-1115.

79.Marques, M., S. Mahapatra, D. Nandan, T. Dick, E.N. Sarno, P. Brennan, and M. Pessolani. 2000. Bacterial and host-derived cationic proteins bind a2-laminins and enhance Mycobacterium leprae attachment to human Schwann cells. Microbes Infect. 2:1407-1417.

80.McGeoch, D.J. 1985. On the predictive recognition of signal peptide sequences. Virus Res. 3:271-286.

81.Mejlhede, N., J.F. Atkins, and J. Neuhard. 1999. Ribosomal -1 frameshifting during decoding of Bacillus subtilis cdd occurs at the sequence CGA AAG. J. Bacteriol. 181:29302937.

82.Moxon, E.R., P.B. Rainey, M.A. Nowak, and R.E. Lenski. 1994. Adaptive evolution of highly mutable loci in pathogenic bacteria. Curr. Biol. 4:24-33.

83.Mushegian, A.R. 2000. Reply to Kyrpides and Ouzounis. Mol. Microbiol. 35:697-698.

84.Nakai, K. 2000. Protein sorting signals and prediction of subcellular localization. Adv. Protein Chem. 54:277-344.

85.Nakai, K. and M. Kanehisa. 1991. Expert system for predicting protein localization sites in gram-negative bacteria. Proteins 11:95-110.

86.Nakashima, H. and K. Nishikawa. 1992. The amino acid composition is different between the cytoplasmic and extracellular sides in membrane proteins. FEBS Lett. 303:141146.

87.Needleman, S.B. and C.D. Wunsch. 1970. A general method applicable to the search for similarities in the amino acid sequence of two proteins. J. Mol. Biol. 48:443-453.

88.Neuwald, A.F., J.S. Liu, and C.E. Lawrence. 1995. Gibbs motif sampling: detection of bacterial outer membrane protein repeats. Protein Sci. 4:1618-1632.

89.Nicholas, H.B., Jr., D.W. Deerfield, II, and A.J. Ropelewski. 2000. Strategies for searching sequence databases. BioTechniques 28:1174-1191.

90.Nielsen, H., S. Brunak, and G. von Heijne. 1999. Machine learning approaches for the prediction of signal peptides and other protein sorting signals. Protein Eng. 12:3-9.

91.Nielsen, H., J. Engelbrecht, S. Brunak, and G. von Heijne. 1997. Identification of prokaryotic and eukaryotic signal peptides and prediction of their cleavage sites. Protein Eng. 10:1-6.

92.Nielsen, H., J. Engelbrecht, S. Brunak, and G. von Heijne. 1997. A neural network method for identification of prokaryotic and eukaryotic signal peptides and prediction of their cleavage sites. Int. J. Neur. Syst. 8:581599.

93.Nilsson, C.L., T. Larsson, E. Gustafsson, K.A. Karlsson, and P. Davidsson. 2000. Identification of protein vaccine candidates from Helicobacter pylori using a preparative two-dimensional electrophoretic procedure and mass spectrometry. Anal. Chem. 72:2148-2153.

94.Nilsson, J., B. Persson, and G. von Heijne. 2000. Consensus predictions of membrane protein topology. FEBS Lett. 486:267-269.

95.Park, S.K., K.I. Kim, K.M. Woo, J.H. Seol, K. Tanaka, A. Ichihara, D.B. Ha, and C.H. Chung. 1993. Site-directed mutagenesis of the dual translational initiation sites of the clpB gene of Escherichia coli and characterization of its gene products. J. Biol. Chem. 268:20170-20174.

96.Parkhill, J., M. Achtman, K.D. James, S.D. Bentley, C. Churcher, S.R. Klee, G. Morelli, D. Basham et al. 2000. Complete DNA sequence of a serogroup A strain of Neisseria meningitidis Z2491. Nature 404:502-506.

97.Pasquier, C., V.J. Promponas, G.A. Palaios, J.S. Hamodrakas, and S.J. Hamodrakas. 1999. A novel method for predicting transmembrane segments in proteins based on a statistical analysis of the SwissProt database: the PRED-TMR algorithm. Protein Eng. 12:381-385.

98.Pautsch, A. and G.E. Schulz. 2000. Highresolution structure of the OmpA membrane domain. J. Mol. Biol. 298:273-282.

99.Pearson, W.R. and D.J. Lipman. 1988. Im proved tools for biological sequence comparison. Proc. Natl. Acad. Sci. USA 85:24442448.

100.Persson, B. 2000. Bioinformatics in protein analysis. Proteomics Funct. Genomics Protein Struct. Anal. 88:215-231.

101.Pizza, M., V. Scarlato, V. Masignani, M.M. Giuliani, B. Arico, M. Comanducci, G.T. Jennings, L. Baldi et al. 2000. Identification of vaccine candidates against serogroup $B$ meningococcus by whole-genome sequencing. Science 287:1816-1820.

102.Rappuoli, R. 2001. Conjugates and reverse 
vaccinology to eliminate bacterial meningitis. Vaccine 19:2319-2322.

103. Rappuoli, R. 2001. Reverse vaccinology, a genome-based approach to vaccine development. Vaccine 19:2688-2691.

104. Rauhut, R. and G. Klug. 1999. mRNA degradation in bacteria. FEMS Microbiol. Rev. 23:353-370.

105. Reinhardt, A. and T. Hubbard. 1998. Using neural networks for prediction of the subcellular location of proteins. Nucleic Acids Res. 26:2230-2236.

106.Richmond, C.S., J.D. Glasner, R. Mau, H. Jin, and F.R. Blattner. 1999. Genome-wide expression profiling in Escherichia coli K12. Nucleic Acids Res. 27:3821-3835.

107.Rigoutsos, I. and A. Floratos. 1998. Com binatorial pattern discovery in biological sequences: The TEIRESIAS algorithm. Bioinformatics 14:55-67.

108.Rost, B., R. Casadio, and P. Fariselli. 1996. Refining neural network predictions for helical transmembrane proteins by dynamic programming. Ismb 4:192-200.

109.Salzberg, S.L., A.L. Delcher, S. Kasif, and O. White. 1998. Microbial gene identification using interpolated Markov models. Nucleic Acids Res. 26:544-548.

110.Saunders, N.J., A.C. Jeffries, J.F. Peden, D.W. Hood, H. Tettelin, R. Rappuoli, and E.R. Moxon. 2000. Repeat-associated phase variable genes in the complete genome sequence of Neisseria meningitidis strain MC58. Mol. Microbiol. 37:207-215.

111.Saunders, N.J., J.F. Peden, D.W. Hood, and E.R. Moxon. 1998. Simple sequence repeats in the Helicobacter pylori genome. Mol. Microbiol. 27:1091-1098.

112.Schirmer, T. and S.W. Cowan. 1993. Prediction of membrane-spanning $\beta$-strands and its application to maltoporin. Protein Sci. 2:1361-1363.

113.Schneider, G. 1999. How many potentially secreted proteins are contained in a bacterial genome? Gene 237:113-121.

114.Seol, J.H., S.J. Yoo, M.S. Kang, D.B. Ha, and C.H. Chung. 1995. The 65-kDa protein derived from the internal translational start site of the clpA gene blocks autodegradation of ClpA by the ATP-dependent protease Ti in Escherichia coli. FEBS Lett. 377:41-43.

115.Shine, J. and L. Dalgarno. 1974. The 3'-terminal sequence of Escherichia coli $16 \mathrm{~S}$ ribosomal RNA: complementarity to nonsense triplets and ribosome binding sites. Proc. Natl. Acad. Sci. USA 71:1342-1346.

116.Shmatkov, A.M., A.A. Melikyan, F.L. Chernousko, and M. Borodovsky. 1999. Finding prokaryotic genes by the "frame-byframe" algorithm: targeting gene starts and overlapping genes. Bioinformatics 15:874886.

117.Smith, R. 2000. Probing proteomes-seeing the whole picture? Nat. Biotechnol. 18:10411042.

118.Smith, T.F. and M.S. Waterman. 1981. Identification of common molecular subsequences. J. Mol. Biol. 147:195-197.

119.Smith, T.F. and X. Zhang. 1997. The challenges of genome sequence annotation or "the devil is in the details". Nat. Biotechnol. 15:1222-1223
120.Sonnhammer, E.L., G. von Heijne, and A. Krogh. 1998. A hidden Markov model for predicting transmembrane helices in protein sequences. Ismb 6:175-182.

121.Spence, J.M. and V.L. Clark. 2000. Role of ribosomal protein L12 in gonococcal invasion of Hec1B cells. Infect. Immun. 68:5002-5010.

122.Stathopoulos, C. 1999. Bacterial outer membrane proteins: topological analyses and biotechnological perspectives. Membr. Cell Biol. 13:3-21.

123.Stephens, R.S. 2000. Chlamydial genomics and vaccine antigen discovery. J. Infect. Dis. 181:S521-S523.

124.Sternberg, N., B. Sauer, R. Hoess, and K. Abremski. 1986. Bacteriophage P1 cre gene and its regulatory region. Evidence for multiple promoters and for regulation by DNA methylation. J. Mol Biol. 187:197-212.

125.Stover, C.K., X.Q. Pham, A.L. Erwin, S.D. Mizoguchi, P. Warrener, M.J. Hickey, F.S. Brinkman, W.O. Hufnagle et al. 2000 Complete genome sequence of Pseudomonas aeruginosa PA01, an opportunistic pathogen. Nature 406:959-964.

126.Sun, Y.-H., S. Bakshi, R. Chalmers, and C.M. Tang. 2000. Functional genomics of Neisseria meningitidis pathogenesis. Nat. Med. 6:1269-1273

127.Sykes, K.F. and S.A. Johnston. 1999. Linear expression elements: a rapid, in vivo, method to screen for gene functions. Nat Biotechnol. 17:355-359.

128.Tateno, Y., S. Miyazaki, M. Ota, H. Sugawara, and T. Gojobori. 2000. DNA data bank of Japan (DDBJ) in collaboration with mass sequencing teams. Nucleic Acids Res. 28:24-26.

129.Tenson, T., J.V. Herrera, P. Kloss, G. Guarneros, and A.S. Mankin. 1999. Inhibition of translation and cell growth by minigene expression. J. Bacteriol. 181:16171622.

130.Tettelin, H., N.J. Saunders, J. Heidelberg, A.C. Jeffries, K.E. Nelson, J.A. Eisen, K.A. Ketchum, D.W. Hood et al. 2000 Complete genome sequence of Neisseria meningitidis serogroup B strain MC58. Science 287:1809-1815.

131.Thomas, G.H. 1999. Completing the E. coli proteome: a database of gene products characterised since the completion of the genome sequence. Bioinformatics 15:860-861

132.Tomb, J.F., O. White, A.R. Kerlavage, R.A. Clayton, G.G. Sutton, R.D. Fleischmann, K.A. Ketchum, H.P. Klenk et al. 1997. The complete genome sequence of the gastric pathogen Helicobacter pylori. Nature 388:539-547.

133.van Belkum, A., S. Scherer, L. van Alphen, and H. Verbrugh. 1998. Short-sequence DNA repeats in prokaryotic genomes. Microbiol. Mol. Biol. Rev. 62:275-293.

134.Venter, J.C., M.D. Adams, E.W. Myers, P.W. Li, R.J. Mural, G.G. Sutton, H.O. Smith, M. Yandell et al. 1304. The sequence of the human genome. Science 291:16.

135.von Heijne, G. 1992. Membrane protein structure prediction. Hydrophobicity analysis and the positive-inside rule. J. Mol. Biol.
225:487-494

136.von Heijne, G. 1986. A new method for predicting signal sequence cleavage sites. Nucleic Acids Res. 14:4683-4690.

137.von Heijne, G. 1985. Signal sequences. The limits of variation. J. Mol. Biol. 184:99-105.

138.von Heijne, G. 1989. The structure of signal peptides from bacterial lipoproteins. Protein Eng. 2:531-534.

139.Weinstock, G.M., D. Smajs, J. Hardham, and S.J. Norris. 2000. From microbial genome sequence to applications. Res. Microbiol. 151:151-158.

140.Wizemann, T.M., J.H. Heinrichs, J.E. Adamou, A.L. Erwin, C. Kunsch, G.H. Choi, S.C. Barash, C.A. Rosen et al. 2001. Use of a whole genome approach to identify vaccine molecules affording protection against Streptococcus pneumoniae infection. Infect. Immun. 69:1593-1598.

141.Wolf, Y.I., I.B. Rogozin, A.S. Kondrashov, and E.V. Koonin. 2001. Genome alignment, evolution of prokaryotic genome organization, and prediction of gene function using genomic context. Genome Res. 11:356-372.

142.Yoshida, K., K. Kobayashi, Y. Miwa, C.M. Kang, M. Matsunaga, H. Yamaguchi, S. Tojo, M. Yamamoto et al. 2001. Combined transcriptome and proteome analysis as a powerful approach to study genes under glucose repression in Bacillus subtilis. Nucleic Acids Res. 29:683-692.

Address correspondence to:

Dr. Robert J. Zagursky

Wyeth-Lederle Vaccines

211 Bailey Road

West Henrietta, NY 14586, USA

e-mail: zagursrj@war.wyeth.com
For reprints of this or any other article, contact Reprints@BioTechniques.com 